\title{
Medical Grade Polyurethane Supported Pseudo Capacitor
}

\author{
Ritu Kataky ${ }^{1}$, Siddhartha Pradeep ${ }^{2}$ \\ ${ }^{1}$ Department of Chemistry, Durham University, Stockton Road, Durham, DH1 3LE \\ ${ }^{2}$ Deparmtnet of Chemical Engineering, Indian Institute of Technology, Hauz Khas, Delhi, 110016
}

\begin{abstract}
An all solid state, thin and flexible redox supercapacitor fabricated by forming layer by layer films using biocompatible materials and then assembling them together, thus making entire supercapacitor thin while also retaining its flexibility. Using these films, this study has also tested the super capacitive properties of Polyvinyl alcohol, graphene oxide(PVA-GO), Polyvinyl alcohol, glucose(PVA-glucose) and Ionic liquids. Electrochemical characterization has demonstrated a good electrical double layer capacitive behavior. The use of non toxic and biocompatible materials along with its miniature size and mechanical flexibility can find its use in many devices under wide range of conditions especially in medical implantable devices.
\end{abstract}

Keywords: Graphene, Medical grade, Super capacitor, Ionic fluids

\section{Introduction}

One of the major fields of innovation in the last few years has been flexible electronic devices. As the gap between microelectronics and biotechnology started to bridge the production of several lifesaving medical implantable devices are emerging [1-2]. Bio microelectronics has diminished size of medical devices to incredible levels and the introduction of nanotechnology to this field has opened up new doors for the next generation of medical implantable devices which can be miniaturized, are robust, biocompatible, portable, wireless possess flexibility, reliability and are economically viable[35].A requirement for these implantable devices is a small and flexible power source with rapid charge and discharge rates. Present day power sources are inadequate as they are made of toxic ingredients, have poor charge/discharge rates, low power density and are also lack mechanical flexibility[5].This is a niche where small electrochemical supercapacitor's can play an important role[6]. Flexible supercapacitor's with rapid charge discharge characteristics are attractive, not only for medical implantable devices but also for flexible, portable, wearable electronics and especially in wireless systems where pulses of short durations is required.

This work builds on previous work where we have shown that Graphene Oxide (GO) incorporated in salinized PVA gels form rolled up graphene oxide nano capsules within the pores of salinized hydrogels may be used as electrochemical pseudo capacitors with physiological glucose or $\mathrm{KOH}$ as a reducing agent, affording a material suitable for devices requiring pulses with characteristic time less than a second [10].

In this paper we have progressed our work by assembling an all solid state, biocompatible supercapacitor device. with excellent, rapid charge-discharge characteristics . GO was incorporated in Tecoflex SG 80 which is a medical grade. Room temperature ionic liquid was added to redox supercapacitor. Electrolytes Glucose and citrate were added to PVA hydrogel to form a gel electrolyte.

\section{Experimental}

Materials were carefully chosen based on their properties especially conductivity and biocompatibility. Graphene oxide (GO) has good electrical conductivity, high young's modulus, large specific area and stability for wide temperature range, it was chosen as one of the ingredient $[7,8]$. Ionic liquids have low vapor pressure, have large electrochemical window, good thermal stability and conductivity and thus was used as electrolyte [9]. PVA also possess desired properties and is also biocompatible, thus it was also included.

All the materials bought were used without any further purification. Materials used were Polyvinyl alcohol (75\% hydrolyzed, Aldrich, UK), Glucose (99.5\%, Aldrich, UK), Tecoflex SG 80 (Lubrizol, Derby, UK ) Tetrahydrofuran (THF), 1-ethyl-3-methyl imidazolium bromide (IL- EMImBr, $97 \%$, Fluka).Graphene oxide was prepared in house by Hummers method.

The device was assembled on a flexible film with a layer of graphite powder (particle size of 40 micrometer) with Platinum wires as electrical connectors were used as electrode bases.

The performance of supercapacitor was checked by Cyclic Voltametry (CV), charge/discharge - short time scale, Electrical impedance spectroscopy (EIS) and dielectric measurement. All the characterizations were done using two electrode configuration in a Faraday cage in order to minimize interference. $\mathrm{CV}$ and Electrical impedance spectroscopy (EIS) were done using potentiostat connected to computer. Analysis was done using software "NOVA" installed on the system. Charge/Discharge measurements were done using IVIUM potentiostat in similar manner. CV measurements were performed between $-0.2 \mathrm{~V}$ and $0.6 \mathrm{~V}$ at different scan rates. EIS measurements were done for frequency varying from 106 and $10-1 \mathrm{~Hz}$ with an amplitude of sinusoidal excitation as $10 \mathrm{mV}$.

This supercapacitor of thickness $2 \mathrm{~mm}$ was fabricated by 


\section{International Journal of Science and Research (IJSR) \\ ISSN (Online): 2319-7064 \\ Index Copernicus Value (2015): 78.96 Impact Factor (2015): 6.391}

sandwiching two halves with a layer of electrolyte gel in between. Each half comprised of comprised of two layers on a flexible thin film of circular $2 \mathrm{~cm}$ diameter.

Composition of each half: Layer 1: 2.86 gram of Tecoflex SG 80 was mixed with $15 \mathrm{ml}$ of THF and the mixture was heated to form a viscous, clear, liquid. This was allowed to cool and $64 \mathrm{mg}$ of graphite powder together with 200 micro liter's IL-EMImBr were added and mixed with the melt. This layer formed the electrode.

Layer 2: this layer was prepared by first dispersing $100 \mathrm{mg}$ dried $\mathrm{GO}$ and 200 micro liter's IL-EMImBrin $15 \mathrm{ml}$ of distilled $\mathrm{H} 2 \mathrm{O}$ and then sonicating it for 4 hours. This dispersion was slowly added to a PVA solution prepared by dissolving $3.25 \mathrm{~g}$ of PVA in $85 \mathrm{ml}$ distilled $\mathrm{H} 2 \mathrm{O}$ at $900 \mathrm{C}$ and allowed to cool to $500 \mathrm{C}$, [10]

The two layers were sandwiched together with a gel electrolyte. The gel electrolyte was made by adding $2 \mathrm{~g}$ of glucose or sodium citrate dehydrate and $75 \mathrm{ml}$ of IL EMImBr to a solution of PVA cooled to 50 0C (Figure 1).

A Pt wire was inserted into the first layer for electrical contact.

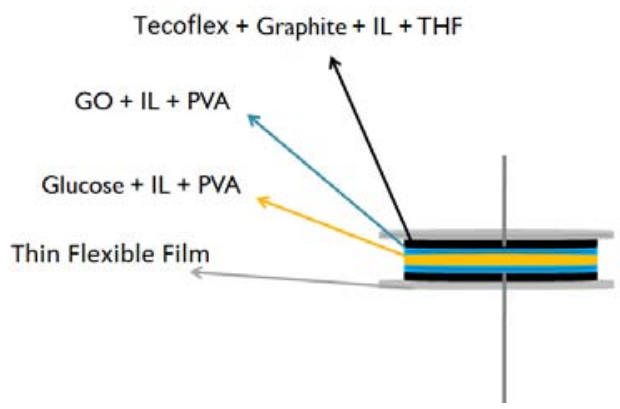

Figure 1: Fabricated supercapacitor with ingredients of different layers

\section{Results \& Discussion}

\subsection{Electrochemical Measurements}

\subsubsection{Cyclic Voltammetry}

$\mathrm{CV}$ was used to calculate the capacitance of supercapacitor which was calculated using equation $(1)^{[11]}$;

$$
C=\frac{1}{2 v\left(V_{f}-V_{t}\right)} \int_{V_{t}}^{V_{f}} I(V) d V
$$

where $\mathrm{C}$ is capacitance in $\mathrm{F}, \mathrm{V}_{\mathrm{f}}$ and $\mathrm{V}_{\mathrm{i}}$ are limits of the potential window in $\mathrm{V}, v$ is the scan rate in $\mathrm{V} \mathrm{s}^{-1}$ and $\mathrm{I}$ is the current in A.

Higher scan rates causes charging discharging at higher power levels. [13, 14] (Figure 2a). Ideal supercapacitor's have rectangular $\mathrm{CV}$ but deviation from this represents presence some psuedocapacitive behavior. . Capacitance calculated using equation (1) was $0.17,0.11,0.07,0.03$, $0 . .02 \mu \mathrm{F} / \mathrm{cm}^{2}$ for scan rates of $20,50,100,250$ and 500 $\mathrm{mVs}-1$ respectively. From capacitance data, it can be observed that with increasing scan rate capacitance decreased which tells us about increasing diffusion resistance for ionic motion into electrode pores at higher sweep rates[13]. Cyclic voltammograms are symmetric or depicts a mirror image, which is a good indication for the selected materials as symmetric voltammograms tells us about the degree reversibility of an electrode reaction [13]. Reversibility adds another advantage for supercapacitor's over rechargeable batteries. These results itself indicate that materials selected and the layers formed have good electrical conductivity and have good storage capabilities and are suitable for use in supercapacitor.

Further, CV measurements done to check the cyclic stability of supercapacitor is shown in (Figure 2b). Calculation of capacitance using equation (1) showed very little change in capacitance between 25th and 50th cycle, representing good cycle life of supercapacitor. Thus it can act as a good energy source for high power instruments. The change in capacitance is attributed to charge consumption due to some faradic reaction with unbounded functional groups at electrode/electrolyte surfaces [16].

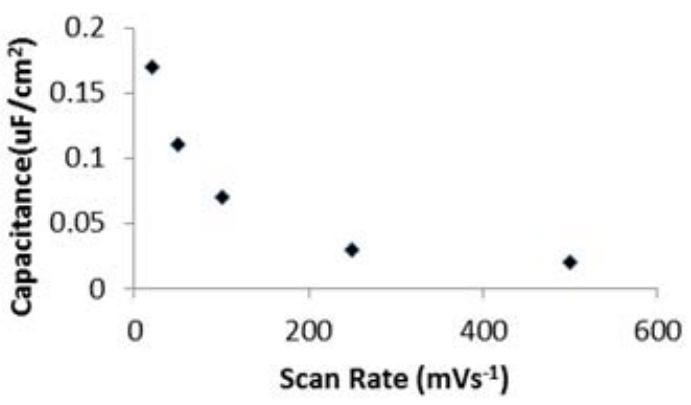

Figure 2a: Variation of capacitance with scan rate

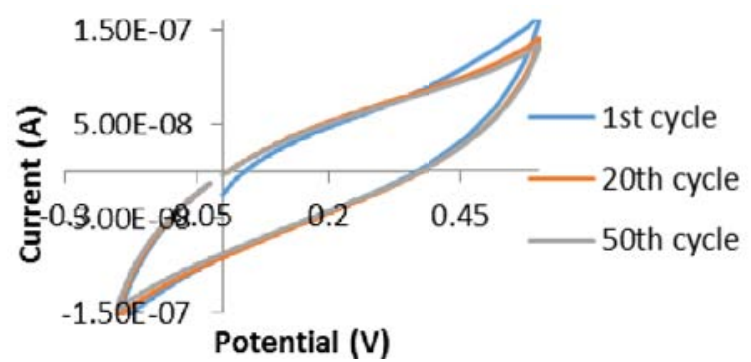

Figure 2b: Cyclic Voltammograms of supercapacitor at scan rate of $250 \mathrm{mV} / \mathrm{s}$ for 50 cycles

\subsubsection{Charge Discharge}

Capacitance was also calculated using Charge Discharge $(\mathrm{C} / \mathrm{D})$ - short time response, using equation $(2)^{[11]}$;

$$
C=I \frac{d t}{d V(t)}
$$

where Cis capacitance in F, $I$ is the charging current, $t$ is the charging time and $\mathrm{V}$ is the change in potential on charge. 

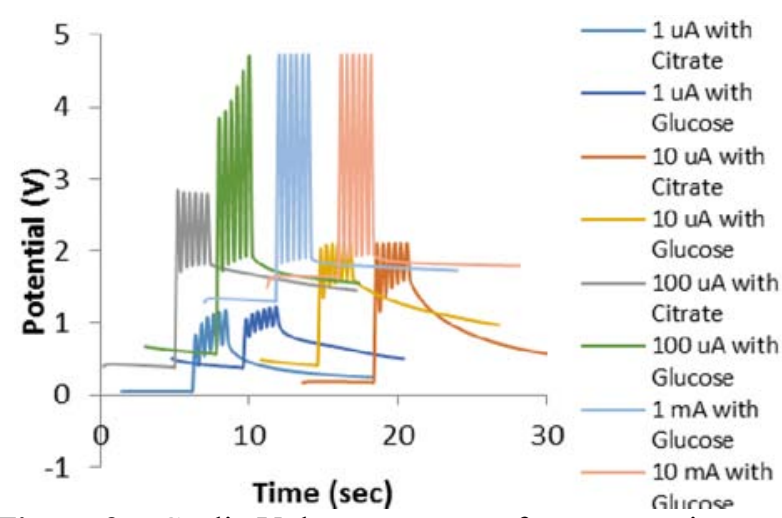

Figure 3a: Cyclic Voltammograms of supercapacitor at scan rate of $250 \mathrm{mV} / \mathrm{s}$ for 50 cycles

Table 1: Avg. Capacitance, charge retention for different charging currents

\begin{tabular}{|c|c|c|}
\hline $\begin{array}{c}\text { Charging current } \\
(\mathrm{mA})\end{array}$ & $\begin{array}{c}\text { Capacitance } \\
(\mu \mathrm{F})\end{array}$ & $\begin{array}{c}\text { Charge Retention } \\
(\%)\end{array}$ \\
\hline 0.001 & 0.5 & 64 \\
\hline .01 & 2.5 & 56 \\
\hline 0.1 & 6.6 & 35 \\
\hline 1 & 88.8 & 13 \\
\hline 10 & 743.4 & 16 \\
\hline
\end{tabular}

Galvanostatic charge Discharge done with short time response (Figure 3a) are displayed for current varying from 1 $\mu \mathrm{A}$ to $10 \mathrm{~mA}$ and working potential window accordingly varying from 1 to $5 \mathrm{~V}$, which is the requirement of many practical wearable equipment's[18,19].. Sharp C/D peaks of these curves open up the possibility of its use in wireless devices where high power is required in short time intervals. Capacitance values for different charging currents were calculated by equation (2) and are given in (Table 1). Charge retention was calculated using the potential change in first charge discharge sequence. Supercapacitor shows great capacitance and decent capacitance retention thus promising charge storage capabilities.

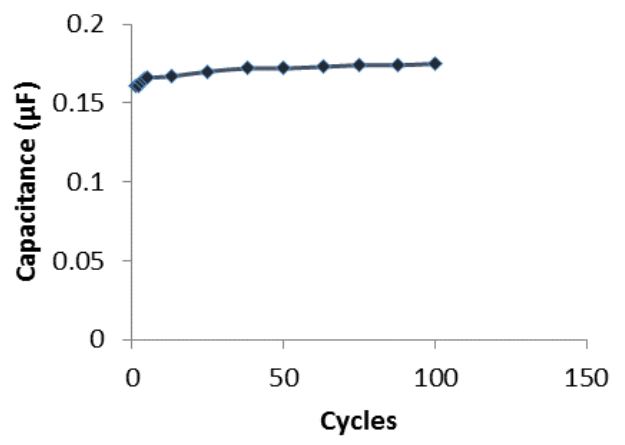

Figure 3b: Change in capacitance with cycles using C/D with $10 \mu \mathrm{A}$ charging current and $0.2 \mathrm{sec}$ intervals for 112 cycles

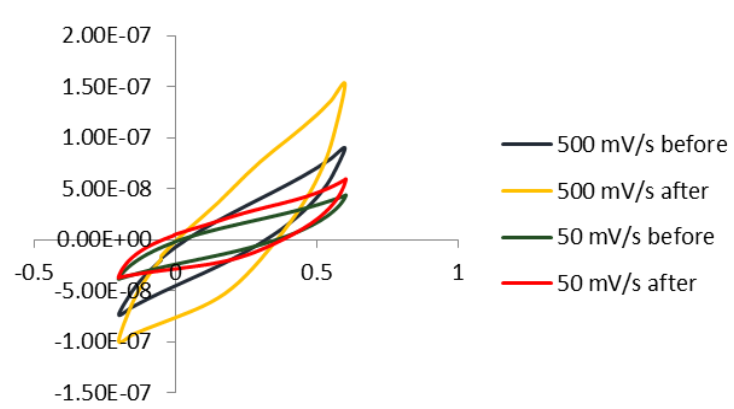

Figure 3c: Cyclic Voltammograms at 500 and $50 \mathrm{mVs}-1$ done before 1 st cycle and after 112 th cycle of C/D to show change in capacitance.

Cyclic stability was also checked from charge discharge curves with charging current of $10 \mu \mathrm{A}$ and intervals of 0.2 sec, (Figure 3a). Plot of calculated capacitances with different cycles (Figure 3b) shows excellent capacitance retention after 120 cycles. It was seen that capacitance actually increased from $0.161 \mu \mathrm{F}$ to $0.175 \mu \mathrm{F}$. This surprising behavior was attributed to electrochemical reduction of RGO. Increment of psuedocapacitive capacitance due to reduction adds to capacitance as was also seen in the experiment performed in [22]. This can also be seen by change of Voltammograms in (Figure 3c). Rise in peak currents and loss of symmetricity along with increase in width of curve also explains the same.

\subsubsection{EIS}

EIS is used to study resistance and capacitance of supercapacitor. EIS data is analysed using Nyquist plots which shows frequency response of system and are a plot of imaginary component of impedance $\left(Z^{\prime \prime}\right)$ against real component of impedance $(Z)$. Every data point on Nyquist plot represents impedance value at different frequency $[7,15,16]$.
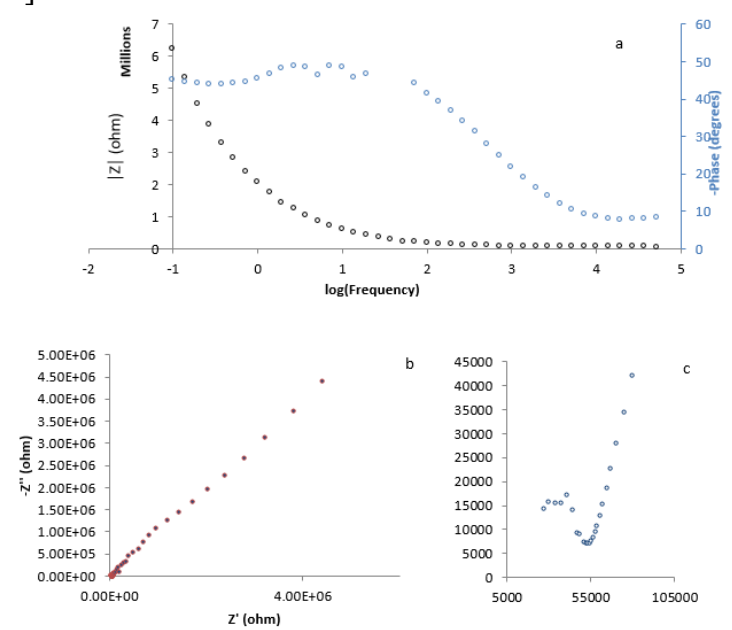

Figure 4: Curves obtained after fitting from EIS data - (a) Bode plots, (b) Nyquist plot for $0.1 \mathrm{~Hz}$ to $10 \mathrm{MHz}$ frequency range (c) Nyquist plot for higher frequencies.

As can be seen from (Figure 4c), Nyquist plot shows an arc in high frequency region and a straight line in low frequency region which tells us about Warburg behavior, which is related to resistance and capacitance of ionic diffusions[16,17]. For ideal capacitor this line is vertical. Because these supercapacitor's are power devices, so they should have low internal resistance thus ESR should be low. 


\section{International Journal of Science and Research (IJSR) \\ ISSN (Online): 2319-7064 \\ Index Copernicus Value (2015): 78.96 Impact Factor (2015): 6.391}

It can seen from (Figure 4a) that on increasing frequency phase angle decreases and at higher frequencies it takes low values, thus showing resistance dominance at high frequencies which is as per expectation. (Figure $4 \mathrm{~b}$ ) can also be used to explain the same thing. At low frequencies, capacitive impedance dominates and high $\mathrm{Z}$ in low frequency represents good capacitance which on increasing frequency reduces to resistive dominant region of higher frequencies. Thus at higher frequencies ions have difficulty to penetrate into the pores, so capacitance decreases and resistance dominates.

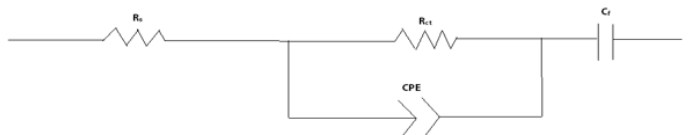

Figure 5: Equivalent circuit model - modified Randels circuit

Equivalent circuit, made from modified Randal's circuit shown (Figure 5), consisting of capacitor along with resistance explains the cause for non ideality and deviations. Rs, resistance in series is the bulk electrolyte resistance, Rct represents the charge transfer resistance, CPE is used for inclusion of non ideality of system, $\mathrm{Cf}$ is included as psuedocapacitance, which is used to account for faradic reactions $[11,20]$.

CPE consists of two parameters, system capacitance CPE-T and CPE-P which accounts for non ideality of system (if it's 1 then system is ideal). CPE can be converted to real capacitance using equation (3);

$$
C=(C P E-T)=0_{\max }[1-(C P E-P)]
$$

where $\omega_{\max }$ is peak frequency of high frequency semi circular region[20,33]. Curve fitting of the plots (ESI Figure 4) was done using Zviewer software which showed sum of squares of deviation, which is a measure of curve fitting as 0.78 which represents good fitting (value less than 1 represents good data fit). Good fitting reveals that this circuit can successfully describe the electrochemical process. Value of CPE-P comes out to be 0.6 which may be due to "limit of ion migration into pores" as also observed by [21] and CPE$\mathrm{T}$ comes out to be 0.12 . $\mathrm{C}_{\mathrm{f}}$ comes out to be $0.35 \mu \mathrm{F}$, confirming some psuedocapacitance. Thus calculating real capacitance with CPE-T as $0.12, \omega_{\max }$ as 350000 and CPE-P as 0.6 we get value of $19.8 \mu \mathrm{F}$.

\section{Conclusion}

In Summary, a good charge storing and flexible supercapacitor was fabricated using ionic liquids, graphene oxide and PVA. CV gave us the highest capacitance of about $0.55 \mu \mathrm{F}$ at $20 \mathrm{mV} \mathrm{s}-1$ and about $0.74 \mathrm{mF}$ at $10 \mathrm{~mA}$ charging current from charge discharge data. Since the potentiostat used for CV changes potential in small steps and the current is measured at the end of the steps during which capacitor relaxes back, due to this current value shown is an underestimate and so is capacitance calculations done by $\mathrm{CV}$ curves. Thus we believe that capacitance calculated by $\mathrm{C} / \mathrm{D}$ is the actual capacitance. Also interesting increase of capacitance was observed with $\mathrm{C} / \mathrm{D}$ due to presence of $\mathrm{RGO}$ which makes it beneficial for supercapacitor's. Also peaks in potential and rapid $\mathrm{C} / \mathrm{D}$ in short response $\mathrm{C} / \mathrm{D}$ measurements ensures it's usage in systems where power demand is time dependent and is generally rapidly. EIS data fitting and calculations showed capacitance of $19.8 \mu \mathrm{F}$ and low internal resistance, thus desirable results were obtained. It's best usage can be with biocompatible pulse devices where large capacitance is not needed whereas rapid response time, low cost and biocompatibility is required[15]. We have just "scratched the surface of the most exiting material of $21 \mathrm{st}$ century" (TIME)[23] - Graphene and more studies about its compounds can provide great lead for supercapacitor development and related devices.

\section{References}

[1] http://mullingsgroup.com/implantable-medical-devicesmarket/, accessed on 27/06/2015

[2] Khan, Wahid, et al. "Implantable medical devices." Focal Controlled Drug Delivery. Springer US, 2014. 33-59.

[3] Genet, Corine, Khalid Errabi, and Caroline Gauthier. "Which model of technology transfer for nanotechnology? A comparison with biotech and microelectronics." Technovation 32.3 (2012): 205-215.

[4] Sayler, Gary S., Michael L. Simpson, and Chris D. Cox. "Emerging foundations: nano-engineering and biomicroelectronics for environmental biotechnology." Current opinion in microbiology 7.3 (2004): 267-273.

[5] Joung, Yeun-Ho. "Development of implantable medical devices: from an engineering perspective." International neurourology journal 17.3 (2013): 98-106.

[6] Meng, Chuizhou, Oren Z. Gall, and Pedro P. Irazoqui. "A flexible super-capacitive solid-state power supply for miniature implantable medical devices."Biomedicalmicrodevices 15.6 (2013): 973-983.

[7] Stoller, Meryl D., et al. "Graphene-based ultracapacitors." Nano letters 8.10 (2008): 3498-3502.

[8] Zhu, Yanwu, et al. "Graphene and graphene oxide: synthesis, properties, and applications." Advanced materials 22.35 (2010): 3906-3924.

[9] Brandt, A., et al. "Ionic liquids in supercapacitors." MRS Bull 38.07 (2013): 554-9.

[10] Kataky, R., et al. "Graphene oxide nanocapsules within silanized hydrogels suitable for electrochemical pseudocapacitors." Chemical Communications(2015).

[11]Joseph Hadden, "Supercapacitor Materials for Implantable devices", 4th year M.Tech Report, Durham University.

[12] Namisnyk, Adam Marcus. A survey of electrochemical supercapacitor technology. Diss. University of Technology, Sydney, 2003.

[13] Andrienko, Denis. "Cyclic Voltammetry." Cyclic Voltammetry (2008).

[14]Bae, Joonho, et al. "Fiber Supercapacitors Made of Nanowire-Fiber Hybrid Structures for Wearable/Flexible Energy Storage." AngewandteChemie International Edition 50.7 (2011): 1683-1687. 
[15]Hossick-Schott, Joachim, John D. Norton, and Craig L. Schmidt. "Capacitor in an implantable medical device." U.S. Patent No. 7,002,790. 21 Feb. 2006.

[16] Hashmi, S. A., Ashok Kumar, and S. K. Tripathi. "Experimental studies on solid state electrical double layer capacitors using activated charcoal powder electrodes and PVdF-HFP based gel electrolytes." Ionics 10.3-4 (2004): 213-220.

[17] Basri, N. H., and B. N. M. Dolah. "Physical and electrochemical properties of supercapacitor electrodes derived from carbon nanotube and biomass carbon."Int. J. Electrochem. Sci 8 (2013): 257-273.

[18] Mallela, Venkateswara Sarma, V. Ilankumaran, and N. Srinivasa Rao. "Trends in cardiac pacemaker batteries." Indian pacing and electrophysiology journal4.4 (2004): 201.

[19] Kenneth A Ellenbogen, Bruce L. Wilkoff, G. N. Kay. C. P. Lau, "Clinical Cardiac Pacing, Defibrillation and Resynchronization Therapy", Elsevier Health Sciences, 18/12/2006, 268.

[20] Ghaemi, M., et al. "Charge storage mechanism of sonochemically prepared $\mathrm{MnO} 2$ as supercapacitor electrode: effects of physisorbed water and proton conduction." ElectrochimicaActa 53.14 (2008): 46074614.

[21]Chen, Wei-Chih, Ten-Chin Wen, and HsishengTeng. "Polyaniline-deposited porous carbon electrode for supercapacitor." ElectrochimicaActa 48.6 (2003): 641649.

[22] Chen, Yao, et al. "High performance supercapacitors based on reduced graphene oxide in aqueous and ionic liquid electrolytes." Carbon 49.2 (2011): 573-580.

[23] http://time.com/3943209/graphene-material-science/, accessed on $4 / 07 / 2015$ 